\title{
A checklist of Norwegian Tardigrada
}

\author{
Terje Meier'
}

Meier T. 2017. A checklist of Norwegian Tardigrada. Fauna norvegica 37: 25-42.

Animals of the phylum Tardigrada are microscopical metazoans that seldom exceed $1 \mathrm{~mm}$ in length. They are recorded from terrestrial, limnic and marine habitats and they have a distribution from Arctic to Antarctica. Tardigrades are also named 'water bears' referring to their 'walk' that resembles a bear's gait. Knowledge of Norwegian tardigrades is fragmented and distributed across numerous sources. Here this information is gathered and validity of some records is discussed. In total 146 different species are recorded from the Norwegian mainland and Svalbard. Among these, 121 species and subspecies are recorded in previous publications and another 25 species are recorded from Norway for the first time.

doi: 10.5324/fn.v37i0.2269. Received: 2017-05-22. Accepted: 2017-12-06. Published online: 2017-12.20. ISSN: 1891-5396 (electronic).

Keywords: Tardigrada, Norway, Svalbard, checklist, taxonomy, literature, biodiversity, new records

1. Prinsdalsfaret 20, NO-1262 Oslo, Norway.

Corresponding author: Terje Meier

E-mail: t-meie@online.no

\section{INTRODUCTION}

The phylum Tardigrada (water bears) currently holds about 1250 valid species and subspecies (Degma et al. 2007, Degma et al. 2017) and are found in a great variety of habitats. They occur in freshwater, marine sediments, algae, mosses, lichens, litter, grassland, cryoconite holes on glaciers and even on barnacles in marine environments (Kristensen \& Hallas 1980). They have a worldwide distribution; from the deep ocean floor to the highest mountains, from Antarctica to the Arctic region (e.g. Ramazzotti \& Maucci 1983). Some species of tardigrades are known to survive extreme conditions in cryptobiosis. An example of extreme survival is Acutuncus antarcticus (Richters 1904) which is recorded to survive frozen for at least 30 years (Tsujimoto et al. 2016). Further information on tardigrade cryptobiosis can be found in Guidetti et al. 2011 and Mjøberg et al. 2011. Some species are predators, but most species feed on plants, plant remains or bacteria.

When observed in a stereomicroscope, live tardigrades resemble small bears walking over the substrate, earning them the popular name 'water bears'. They have cylindrical bodies with four pairs of stumpy, unsegmented legs usually terminating in claws or sucking disks. The first three pairs of legs are directed ventrolaterally and are used to moving over the substrate. The hind legs are directed posteriorly and are used for grasping. Adult Tardigrades usually range from $250 \mu \mathrm{m}$ to 700 $\mu \mathrm{m}$ in length. Newly hatched juveniles might be as small as 50 $\mu \mathrm{m}$ (Ramazzotti \& Maucci 1983) and mature adults as large as $1200 \mu \mathrm{m}$ (Rahm 1931). In general limnoterrestrial tardigrades of the class Eutardigrada are transparent, while the terrestrial, armour-plated class Heterotardigrada are opaque. They occur in different colours: red, brown, orange, yellow, pink, green or black in the cuticule or gut (Nelson 2002). Reproduction is either sexual or parthenogenetic (Bertolani 1987) and the eggs are laid freely or in the exuvia.

Tardigrades were first described by the German pastor Johann August Ephraim Goeze in 1773. In 1776 the Italian priest and scientist Lazzaro Spallanzani named them Tardigrada, which mean "slow walker" (Spallanzani 1776). The first tardigrade species to be properly described was Macrobiotus hufelandi Schulze, 1834 and Milnesium tardigradum Doyère (1840). These two species were for many years considered to be cosmopolitian and were recorded in many faunistic and other studies from various localities worldwide (Ramazzotti 
Table I. Tardigrada species described from Norway.

\begin{tabular}{|c|c|c|c|}
\hline Taxon & Species & Author & Type locality \\
\hline \multicolumn{4}{|c|}{ HETEROTARDIGRADA } \\
\hline \multicolumn{4}{|c|}{ Echiniscidae } \\
\hline & Bryodelphax parvuspolaris & Kaczmarek et al., 2012 & Revdalen (Svalbard) \\
\hline & Diploechiniscus oihonnae & (Richters, 1903b) & $\begin{array}{l}\text { Meråker (Nord-Trøndelag) } \\
\text { Smeerenburg (Svalbard) }\end{array}$ \\
\hline & Echiniscus blumi & Richters, 1903b & Billefjorden (Svalbard) \\
\hline & Echiniscus loxophthalmus & Richters, 1911a & Tromsø (Troms) \\
\hline & Echiniscus merokensis & Richters, 1903b & $\begin{array}{l}\text { Meråker (Nord-Trøndelag) } \\
\text { Smeerenburg (Svalbard) }\end{array}$ \\
\hline & Echiniscus wendti & Richters, 1903b & $\begin{array}{l}\text { Meråker (Nord-Trøndelag) } \\
\text { Smeerenburg (Svalbard) }\end{array}$ \\
\hline & Echiniscus spiculifer & Schaudinn, 1901 & Storøya (Svalbard) \\
\hline & Testechiniscus spitsbergensis & (Scourfield, 1897) & Advent Bay (Svalbard) \\
\hline
\end{tabular}

\section{EUTARDIGRADA}

\section{APOCHELA}

Milnesiidae

Bergtrollus dzimbowski

Dastych, 2011

Lyngsalpene (Troms)

Bertolanius nebulosus

(Dastych, 1983)

Atomfjella (Svalbard)

\section{PARACHELA}

Eohypsibiidae

Bertolanius volubilis

(Durante Pasa et al., 1975)

Forså (Nordland)

Hypsibiidae

Diphascon stappersi
Hypsibius arcticus
Adropion belgicae
Itaquascon enckelli
Mesocrista spitzbergensis
Pilatobiotus recamieri

Richters, 1911b

Murray, 1907

(Richters, 1911b)

(Mihelčič, 1971/1972)

(Richters, 1903b)

(Richters, 1911b)

Isohypsibiidae

Doryphoribius macrodon

Halobiotus stenostomus

Isohypsibius ceciliae

Isohypsibius coulsoni

Isohypsibius karenae

Isohypsibius pilatoi

Isohypsibius pulcher
Binda et al., 1980

(Richters, 1908)

Pilato \& Binda, 1987

Kaczmarek et al., 2012

Zawierucha, 2013

Durante Pasa \& Maucci, 1977

Mihelčič, 1971/1972
Advent Bay (Svalbard); Hopen

Prins Karls Forland (Svalbard)

Franz Josef Land (Russia)

Advent Bay (Svalbard); Hopen

Alta (Finnmark)

Erkenbäcken (Sverige)

Kenesjärvi; Saima-Puumala (Finland)

Smeerenburg (Svalbard)

Advent Bay; Hopen (Svalbard)

Cap Hold with the Hope (Greenland)

Atomfjella (Svalbard)

Adrono (Sicilia, Italy)

Sydney (Austalia)

Indrepollen (Hordaland)

Magdalenefjorden (Svalbard)

Revdalen (Svalbard)

Ariekammen (Svalbard)

Kvalsund (Finnmark)

Suså (Denmark)

Lakselv (Finnmark)

Mörrumsån; Piteelveen; Kalix (Sweden) 
Table I. Continued.

\begin{tabular}{|c|c|c|c|}
\hline Taxon & Species & Author & Type locality \\
\hline \multicolumn{4}{|c|}{ Macrobiotidae } \\
\hline & Macrobiotus ariekammensis & Węglarska, 1965 & Ariekammen (Svalbard) \\
\hline & Macrobiotus crenulatus & Richters, $1903 b$ & Smeerenburg (Svalbard) \\
\hline & Macrobiotus echinogenitus & Richters, $1903 \mathrm{~b}$ & $\begin{array}{l}\text { Smeerenburg (Svalbard) } \\
\text { Taunus; Hallgarter Zane; Weilburg; Lahn } \\
\text { (Germany) }\end{array}$ \\
\hline & Macrobiotus norvegicus & Mihelčič, 1971/1972 & Kuvatn (Finmark) \\
\hline & $\begin{array}{l}\text { Mesobiotus harmsworthi } \\
\text { harmsworthi }\end{array}$ & (Murray, 1907) & $\begin{array}{l}\text { Prins Karls Forland (Svalbard) } \\
\text { Franz Josef Land (Russia) }\end{array}$ \\
\hline & $\begin{array}{l}\text { Mesobiotus harmsworthi } \\
\text { obscurus }\end{array}$ & (Dastych, 1985) & $\begin{array}{l}\text { Hornsund; Albert I Land; Andreé Land; } \\
\text { Atomfjella (Svalbard) }\end{array}$ \\
\hline & Paramacrobiotus areolatus & (Murray, 1907) & $\begin{array}{l}\text { Prins Karls Forland (Svalbard) } \\
\text { Franz Josef Land (Russia) }\end{array}$ \\
\hline & Tenuibiotus voronkovi & (Tumanov, 2007) & Kongsfjorden (Svalbard) \\
\hline \multicolumn{4}{|c|}{ Richtersiidae } \\
\hline & Adorybiotus granulatus & (Richters, 1903b) & Meråker (Nord-Trøndelag) \\
\hline & Richtersius coronifer & (Richters, 1903b) & $\begin{array}{l}\text { Billefjorden (Svalbard) } \\
\text { Troms } \varnothing \text { (Troms) }\end{array}$ \\
\hline
\end{tabular}

\& Maucci 1983; Tumanov 2006). As new taxonomical tools and traits were added to the identification toolbox in the recent years, more species could be separated and both species are now considered members of more complex species groups (Pilato 1981; Maucci 1988(1991); Binda \& Pilato 1990). For instance the genus Milnesium now contains more than thirty different species (Morek et al., 2016; Pilato et al., 2016).

The first record of tardigrades from Norway was made by Goës (1862) who reported Hypsibius dujardini (Doyère 1840) from Smeerenburg on Svalbard. At the end of the $19^{\text {th }}$ century, Scourfield (1897) reported four species of tardigrades from Svalbard. Among them the common and widespread species Echiniscus spitsbergensis (now: Testechiniscus s.), which he described as new. The following year Richard (1898) found Macrobiotus macronyx (Dujardin 1851 (now: Dactylobiotus)) on the archipelago. Schaudinn (1901) added another seven species to the list of known tardigrades from Svalbard. Pioneers on tardigrade research in the first decade of the $20^{\text {th }}$ century were the German zoologist Ferdinand Richters and the Scottish biologist James Murray. They both contributed to the knowledge of the Norwegian tardigrade fauna (e.g. Richters 1903, 1904a,b, 1908, 1911a,b and Murray 1907). After this period 58 years elapsed before tardigrades from Norway again were mentioned in literature. The two Polish zoologist, Weglarska (1965) and Dastych (1985) published thorough reports about the tardigrade fauna on Svalbard. Studies of tardgrades from limnic biotopes have hitherto been scarcely reported. Mihelčič (1971, 1971/1972) investigated 426 limnic samples from Scandinavia, Iceland and Finland, whereof 69 Norwegian samples revealed eight different species. Back in 1974 Durante Pasa and Maucci $(1975,1979)$ collected 204 samples of moss and lichens from different localities in Norway and their investigation revealed 49 different species. A peculiar species within the family Milnesiidae from Lyngsalpene (Troms) was described by Dastych in 2011. The conspicuously proboscis formed by the mouth region made Dastych establish a new genus for Bergtrollus dzimbowski Dastych, 2011.

The main focus of tardigrades from Norway has been on Svalbard and in recent years many articles have focused on taxonomy of tardigrades from the archipelago. (e.g. Binda et al. 1980, Coulson et al., 2014; Dastych 1983, 1985; De Smet and van Rompu 1994; De Smet, Van Rompu and Beyens 1987, 1988; Janiec 1996; Kaczmarek et al. 2012; Klekowski and Opalinski 1986, 1989; Maucci 1996; Mokievsky 1992; Pilato et al., 1982; Pilato and Binda 1987; Smykla et al. 2011; Tumanov 2007; Van Rompu and De Smet 1988, 1991, 1996; Jankovská et al., 2016; Vonnahme et al., 2016; Zawierucha 2013; Zawierucha et al., 2013, 2015a,b,c; 2016a,b,c,d; 2017).

Among all the recorded species from Norway, 34 are originally described as new to science. Thirteen of these were not linked to a specific type locality (Table 1), but reported from two or more localities in the original description. In many cases type material is lost and the designation of neotypes from type localities might be necessary to stabilize the nomenclature and future understanding of these species.

\section{MATERIAL AND METHODS}

The current list of Tardigrada species (Table 2) is assembled based on recorded species from 60 papers dealing with tardigrades from the Norwegian mainland and Svalbard. All species listed are valid in «Actual checklist of Tardigrada 
Table 2. Tardigrada species recorded from Norway ( ${ }^{*}$ New additions to the species list recorded by the author). Abbreviations for Counties: $\mathrm{AA}=$ Aust-Agder; Ak = Akershus; $\mathrm{Bu}=$ Buskerud; Fi = Finnmark; He = Hedmark; Ho = Hordaland; No = Nordland; NT = Nord-Trøndelag; Op = Oppland; Oslo; Ro = Rogaland; SF = Sogn og Fjordane; Sv = Svalbard; ST = Sør-Trøndelag; Te = Telemark; Tr = Troms; VA = VestAgder; Vf = Vestfold; Øf = Østfold.

\begin{tabular}{|c|c|c|c|c|}
\hline Taxon & Species & Authority & County & Reference \\
\hline \multicolumn{5}{|c|}{ HETEROTARDIGRADA } \\
\hline \multicolumn{5}{|c|}{ ARTHROTARDIGRADA } \\
\hline \multicolumn{5}{|c|}{ Batillipedidae } \\
\hline & Batillipes mirus & Richters, 1909 & Ho & Thambs-Lyche 1940 \\
\hline \multicolumn{5}{|c|}{ Tanarctidae } \\
\hline & $\begin{array}{l}\text { Actinarctus doryphorus } \\
\text { doryphorus }\end{array}$ & Schulz, 1935 & Ho & Clausen 2004 \\
\hline \multicolumn{5}{|c|}{ ECHINISCOIDEA } \\
\hline \multicolumn{5}{|c|}{ Echiniscoididae } \\
\hline & $\begin{array}{l}\text { Echiniscoides sigismundi } \\
\text { groenlandicus }\end{array}$ & $\begin{array}{l}\text { Kristensen \& } \\
\text { Hallas, } 1980\end{array}$ & $\operatorname{Tr}$ & Kristensen \& Hallas 1980 \\
\hline & $\begin{array}{l}\text { Echiniscoides sigismundi } \\
\text { sigismundi }\end{array}$ & (M. Schultze, 1865) & Ho, Oslo & $\begin{array}{l}\text { Richters 1908; Thambs-Lyche 1940; } \\
\text { Kristensen \& Hallas } 1980\end{array}$ \\
\hline \multicolumn{5}{|c|}{ Echiniscidae } \\
\hline & Acanthechiniscus islandicus & (Richters, 1904) & $\mathrm{Sv}$ & Van Rompu \& De Smet 1996 \\
\hline & Acanthechiniscus victor & (Ehrenberg, 1853) & $\mathrm{Sv}$ & $\begin{array}{l}\text { Schaudinn 1901; Richters 1911a; } \\
\text { Marcus 1936; Węglarska 1965; } \\
\text { Dastych 1985; Lagisz 1999; } \\
\text { Zawierucha et al. 2015c; Vonnahme } \\
\text { et al. } 2016\end{array}$ \\
\hline & Bryodelphax parvulus & Thulin, 1928 & Sv, No & $\begin{array}{l}\text { Durante-Pasa \& Maucci 1979; } \\
\text { Łagisz 1999; Zawierucha et al. } \\
\text { 2015c }\end{array}$ \\
\hline & Bryodelphax parvuspolaris & $\begin{array}{l}\text { Kaczmarek et al., } \\
2012\end{array}$ & $\mathrm{~Sv}$ & Kaczmarek et al. 2012 \\
\hline & Bryodelphax sinensis & (Pilato, 1974) & $\mathrm{Sv}$ & Dastych 1985 \\
\hline & Diploechiniscus oihonnae & (Richters, 1903) & $\begin{array}{l}\text { Sv, No, NT, } \\
\text { Ho }\end{array}$ & $\begin{array}{l}\text { Richters 1903, 1904a,b, 1911a,b; } \\
\text { Durante Pasa \& Maucci } 1979\end{array}$ \\
\hline & Echiniscus arctomys & Ehrenberg, 1853 & Sv, No, NT & $\begin{array}{l}\text { Scourfield 1897; Schaudinn 1901; } \\
\text { Richters 1903, 1904b; Murray 1907; } \\
\text { Summerhayes \& Elton 1923; Marcus } \\
\text { 1936; Durante Pasa \& Maucci } 1979\end{array}$ \\
\hline & Echiniscus blumi blumi & Richters, 1903 & Sv, No, NT & $\begin{array}{l}\text { Richters 1903, 1904b; Durante Pasa } \\
\text { \& Maucci 1975, 1979; Dastych } \\
\text { 1985; Maucci 1996; Lagisz 1999; } \\
\text { Zawierucha et al. 2015c }\end{array}$ \\
\hline & Echiniscus capillatus & Ramazzotti, 1956 & $\mathrm{~Sv}$ & $\begin{array}{l}\text { Dastych 1985; Lagisz 1999; } \\
\text { Zawierucha et al. 2015c }\end{array}$ \\
\hline & Echiniscus columinis & Murray, 1911 & Sv & Węglarska 1965 \\
\hline & Echiniscus granulatus & (Doyère, 1840) & Sv, No & $\begin{array}{l}\text { Richters 1911a; Durante Pasa } \\
\text { \& Maucci 1975, 1979; Dastych } \\
\text { 1985; Maucci 1996; Łagisz 1999; } \\
\text { Zawierucha et al. 2016b }\end{array}$ \\
\hline & Echiniscus loxophthalmus & Richters, 1911 & $\operatorname{Tr}$ & Richters 1911a \\
\hline
\end{tabular}


Table 2. Continued.

\begin{tabular}{|c|c|c|c|c|}
\hline Taxon & Species & Authority & County & Reference \\
\hline & $\begin{array}{l}\text { Echiniscus merokensis } \\
\text { merokensis }\end{array}$ & Richters, 1904 & $\begin{array}{l}\text { Sv, Fi, Tr, } \\
\text { No, NT, ST, } \\
\text { OP }\end{array}$ & $\begin{array}{l}\text { Richters 1904b; Marcus 1936; } \\
\text { Durante Pasa \& Maucci 1979; } \\
\text { Dastych 1985; Maucci 1996; Meier } \\
\text { 1996; Kaczmarek et al. 2012; } \\
\text { Zawierucha et al. 2013, 2015c, } \\
\text { 2016b, } 2017\end{array}$ \\
\hline & $\begin{array}{l}\text { Echiniscus merokensis } \\
\text { suecicus }\end{array}$ & Thulin, 1911 & $\mathrm{~Sv}$ & Maucci 1996 \\
\hline & Echiniscus militaris & Murray, 1911 & No & Durante Pasa \& Maucci 1975, 1979 \\
\hline & Echiniscus phocae & $\begin{array}{l}\text { du Bois-Reymond } \\
\text { Marcus, } 1944\end{array}$ & No & Durante Pasa \& Maucci 1979 \\
\hline & $\begin{array}{l}\text { Echiniscus quadrispinosus } \\
\text { quadrispinosus }\end{array}$ & Richters, 1902 & $\mathrm{~Sv}$ & $\begin{array}{l}\text { Marcus 1936; Kaczmarek et al. } \\
\text { 2012; Zawierucha et al. 2016b }\end{array}$ \\
\hline & Echiniscus spiculifer & Schaudinn, 1901 & $\mathrm{~Sv}$ & Schaudinn 1901; Marcus 1936 \\
\hline & Echiniscus spinulosus & (Doyère, 1840) & Sv & Schaudinn 1901; Marcus 1936 \\
\hline & Echiniscus testudo & (Doyère, 1840) & Sv, No & $\begin{array}{l}\text { Schaudinn 1901; Richters 1903, } \\
\text { 1904b; Summerhayes \& Elton } \\
\text { 1923; Marcus 1936; Durante Pasa \& } \\
\text { Maucci 1979; Dastych 1985; Maucci } \\
\text { 1996; Kaczmarek et al. 2012; } \\
\text { Zawierucha et al. 2016b }\end{array}$ \\
\hline & Echiniscus trisetosus & Cuénot, 1932 & Sv, No, Op & $\begin{array}{l}\text { Marcus 1936; Durante Pasa \& } \\
\text { Maucci 1975, } 1979\end{array}$ \\
\hline & Echiniscus wendti & Richters, 1903 & $\begin{array}{l}\text { Sv, Tr, NT, } \\
\text { Op }\end{array}$ & $\begin{array}{l}\text { Richters 1903, 1904b, 1911a,b; } \\
\text { Węglarska 1965; Durante Pasa \& } \\
\text { Maucci 1979; Dastych 1985, 2011; } \\
\text { Janiec 1996; Maucci 1996; Lagisz } \\
\text { 1999; Kaczmarek et al. 2012; } \\
\text { Zawierucha et al. 2015c, 2016b }\end{array}$ \\
\hline & $\begin{array}{l}\text { Hypechiniscus gladiator } \\
\text { gladiator }\end{array}$ & (Murray, 1905) & ST & $\begin{array}{l}\text { *Meier, Mølnbukt (Agdenes, Sør- } \\
\text { Trøndelag) }\end{array}$ \\
\hline & Proechiniscus hanneae & (Petersen, 1951) & $\operatorname{Tr}$ & Unpublished (Dastych - pers. med.) \\
\hline & Pseudechiniscus suillus & (Ehrenberg, 1853) & $\begin{array}{l}\text { Sv, No, NT, } \\
\text { ST, Op }\end{array}$ & $\begin{array}{l}\text { Murray 1907; Richters 1911b; } \\
\text { Marcus 1936; Węglarska 1965; } \\
\text { Durante Pasa \& Maucci 1975, } \\
\text { 1979; Dastych 1985; Van Rompu } \\
\text { \& De Smet 1991, 1996; Meier 1996; } \\
\text { Maucci 1996; Kaczmarek et al. } \\
\text { 2012; Zawierucha et al. 2013, 2015c }\end{array}$ \\
\hline & $\begin{array}{l}\text { Testechiniscus spitsber- } \\
\text { gensis }\end{array}$ & (Scourfield, 1897) & Sv, No & $\begin{array}{l}\text { Scourfield 1897; Schaudinn 1901; } \\
\text { Richters 1903, 1904b, 1911a,b; } \\
\text { Marcus 1936; Węglarska 1965; } \\
\text { Durante Pasa \& Maucci 1975, 1979; } \\
\text { Dastych 1985; Van Rompu \& De } \\
\text { Smet 1991, 1996; Maucci 1996; } \\
\text { Kaczmarek et al. 2012; Zawierucha } \\
\text { et al. 2013, 2015c; Vonnahme et } \\
\text { al. } 2016\end{array}$ \\
\hline
\end{tabular}

\section{EUTARDIGRADA}

APOCHELA

Milnesiidae 
Table 2. Continued.

\begin{tabular}{|c|c|c|c|c|}
\hline Taxon & Species & Authority & County & Reference \\
\hline & Milnesium asiaticum & Tumanov, 2006 & $\mathrm{~Sv}$ & $\begin{array}{l}\text { Kaczmarek et al. 2012; Zawierucha } \\
\text { et al. } 2015 \text { c }\end{array}$ \\
\hline & Milnesium eurystomum & Maucci, 1991 & Sv, Tr & $\begin{array}{l}\text { Dastych 2011; Kaczmarek et al. } \\
\text { 2012; Zawierucha et al. 2015c }\end{array}$ \\
\hline & $\begin{array}{l}\text { Milnesium tardigradum } \\
\text { tardigradum }\end{array}$ & Doyère, 1840 & $\begin{array}{l}\text { Sv, Fi, Tr, } \\
\text { No, NT, Op, } \\
\text { Øf }\end{array}$ & $\begin{array}{l}\text { Richters 1903, 1904b, 1911a,b; } \\
\text { Marcus 1936; Węglarska 1965; } \\
\text { Durante Pasa \& Maucci 1975, 1979; } \\
\text { Maucci 1996; Łagisz 1999; Dastych } \\
\text { 2011: cf. tardigradum }\end{array}$ \\
\hline
\end{tabular}

\section{Eohypsibioidea}

Eohypsibiidae

$\begin{array}{lll}\text { Bertolanius nebulosus } & \text { (Dastych, 1983) } & \text { Sv, ST } \\ \text { Bertolanius smreczynskii } & \text { (Węglarska, 1970) } & \text { Sv } \\ \text { Bertolanius volubilis } & \begin{array}{l}\text { (Durante Pasa \& } \\ \text { Maucci, 1975) }\end{array} & \text { No, ST } \\ \text { Bertolanius weglarskae } & \text { (Dastych, 1972) } & \text { Sv, ST }\end{array}$

Hypsibioidea

Calohypsibiidae

Calohypsibius ornatus

Calohypsibius schusteri

Calohypsibius verrucosus

Hypsibiidae

Diphasconinae

$\begin{array}{lll}\text { Diphascon alpinum } & \text { Murray, 1906 } & \text { Sv, Fi, Tr, } \\ \text { No, NT, } \\ & & \\ \text { Diphascon chilenense } & \text { Plate, 1888 } & \text { Sv, Tr } \\ \text { Diphascon higginsi } & \text { Binda, 1971 } & \text { ST } \\ \text { Diphascon pingue pingue } & \text { (Marcus, 1936) } & \text { Sv }\end{array}$

Diphascon stappersi

Diphascon tenue $\begin{array}{ll}\text { (Richters, 1900) } & \text { Sv, Tr, No, } \\ \text { NT, Op }\end{array}$

Nelson \&

McGlothlin, 1996

(Richters, 1900)

ST

ST

$\mathrm{Sv}, \operatorname{Tr}$

SV

Richters, $1911 \quad$ Sv

Thulin, 1928 Sv, Tr, ST
Durante Pasa \& Maucci 1979 (see Maucci 1996); Dastych 1983, 1985; Meier 1996

Klekowski \& Opaliński 1986, 1989; De Smet et al. 1988; Janiec 1996

Durante Pasa \& Maucci 1975, 1979; Meier 1996

Van Rompu \& De Smet 1988, 1991, 1996; Meier 1996

Schaudinn 1901; Richters 1903, 1904b, 1911a,b; Murray 1907; Durante Pasa \& Maucci 1979; Kaczmarek et al. 2012; Zawierucha et al. 2015c

*Meier, Mølnbukt (Agdenes, SørTrøndelag)

*Meier, Verrafjorden (Agdenes, SørTrøndelag)

Murray 1907; Marcus 1936; Węglarska 1965; Durante Pasa \& Maucci 1979; De Smet et al. 1988; Van Rompu \& De Smet 1988; Janiec 1996; Łagisz 1999

Richters 1903, 1904b, 1911a,b; Murray 1907; Marcus 1936

*Meier, Søndre Knutshø (Oppdal, Sør-Trøndelag)

Dastych 1985; Van Rompu \& De Smet 1991, 1996; Maucci 1996; Kaczmarek et al. 2012; Zawierucha et al. 2015c, 2016b, 2017

Richters 1911a,b; Marcus 1936; Van Rompu \& De Smet 1991

Węglarska 1965; Durante Pasa \& Maucci 1979; Dastych 1985; Maucci 1996; Meier 1996; Zawierucha et al. $2015 \mathrm{c}$ 
Table 2. Continued.

\begin{tabular}{|c|c|c|c|c|}
\hline Taxon & Species & Authority & County & Reference \\
\hline \multicolumn{5}{|c|}{ Hypsibiinae } \\
\hline & Borealibius zetlandicus & (Murray, 1907) & $\begin{array}{l}\mathrm{Sv}, \mathrm{Fi}, \mathrm{ST} \\
\mathrm{He}, \mathrm{Op}, \mathrm{Bu}\end{array}$ & $\begin{array}{l}\text { Murray 1907; Marcus 1936; Meier } \\
\text { 1996; Lagisz } 1999\end{array}$ \\
\hline & Hypsibius arcticus & (Murray, 1907) & $\mathrm{Sv}$ & $\begin{array}{l}\text { Murray 1907; Richters 1911b; } \\
\text { Marcus 1936; Węglarska 1965; } \\
\text { Dastych 1985: cf. arcticus; Van } \\
\text { Rompu \& De Smet 1991; Janiec } \\
\text { 1996; Zawierucha et al. 2015b,c; } \\
\text { Vonnahme et al. } 2016\end{array}$ \\
\hline & Hypsibius biscuitiformis & Bartoš, 1960 & $\mathrm{~Sv}$ & $\begin{array}{l}\text { Łagisz } 1999 \text { (uncertain identification } \\
\text { according to the author) }\end{array}$ \\
\hline & Hypsibius convergens & (Urbanowicz, 1925) & $\begin{array}{l}\text { Sv, Fi, Tr, No, } \\
\text { NT }\end{array}$ & $\begin{array}{l}\text { Murray 1907; Marcus } 1936 \text { (uncer- } \\
\text { tain); Węglarska 1965; Mihelčič } \\
\text { 1971; Durante Pasa \& Maucci 1975, } \\
\text { 1979; Dastych 1985; Van Rompu } \\
\text { \& De Smet 1991; Janiec 1996; } \\
\text { Łagisz 1999; Kaczmarek et al. 2012; } \\
\text { Zawierucha et al. 2013, 2016b }\end{array}$ \\
\hline
\end{tabular}

Hypsibius dujardini

Hypsibius microps

Hypsibius pallidoides

Hypsibius pallidus

Hypsibius scabropygus

Itaquasconinae
Sv, Fi, ST, Goës 1862; Richters 1903, 1904b, $\mathrm{Op}, \mathrm{Bu}$

1911a,b; Marcus 1936; Węglarska
(Doyère, 1840)

Thulin, 1928

Pilato et al., 2011

Thulin, 1911

Cuénot, 1929

Vf

(Thulin, 1928)

(Dastych, 1987)

Sv

Sv

(Richters, 1911)

$\mathrm{Sv}, \mathrm{Bu}$

ST 1965; Dastych 1985; De Smet et al. 1987, 1988; Van Rompu \& De Smet 1988, 1991, 1996; Janiec 1996; Maucci 1996; Meier 1996; Zawierucha et al. 2013, 2015b, 2016a,b,d, 2017; Vonnahme et al. 2016

Sv, Fi, Tr, No, Durante Pasa \& Maucci 1975, 1979; Øf Maucci 1996; Kaczmarek et al. 2012; Zawierucha et al. 2015c

*Meier, Mølnbukt (Agdenes, SørTrøndelag)

Sv, Fi, Tr, No, Węglarska 1965; Durante Pasa \& ST, Op, Øf $\quad$ Maucci 1979; Dastych 1985; Janiec 1996; Maucci 1996; Meier 1996; Kaczmarek et al. 2012; Zawierucha et al. 2015c, 2016b

*Meier, Havna (Tjøme, Vestfold)

Dastych 1985

Adropion behanae

Adropion belgicae

Adropion prorsirostre

(Thulin, 1928)

Sv, Tr, No, $\mathrm{Op}$

Dastych 1985, 1987

Richters 1911a,b; Marcus 1936; Mihelčič 1971; Dastych 1985; Maucci 1996; Zawierucha et al. 2015c

Maucci 1996; Kaczmarek et al. 2012;

Zawierucha et al. 2013, 2015c, 2017

Adropion scoticum scoticum (Murray, 1905)
Sv, Fi, No, Murray 1907; Richters 1911b; ST, Op, Øf Węglarska 1965; Durante Pasa Maucci 1979; Dastych 1985; De Smet et al. 1988; Van Rompu \& De Smet 1991, 1996; Janiec 1996; Meier 1996; Łagisz 1999; Kaczmarek et al. 2012; Zawierucha et al. 2015c, 2017 
Table 2. Continued.

\begin{tabular}{|c|c|c|c|c|}
\hline Taxon & Species & Authority & County & Reference \\
\hline & Astatumen trinacriae & (Arcidiacono, 1962) & Op & *Meier, Eid (Gran, Oppland) \\
\hline & Itaquascon enckelli & $\begin{array}{l}\text { (Mihelčič, 1971/ } \\
\text { 1972) }\end{array}$ & $\mathrm{Fi}$ & Mihelčič 1971, 1971/1972 \\
\hline & Mesocrista spitzbergensis & (Richters, 1903) & $\begin{array}{l}\text { Sv, Fi, Tr, No, } \\
\text { Op }\end{array}$ & $\begin{array}{l}\text { Richters 1903, 1904b, 1911b; Murray } \\
\text { 1907; Marcus 1936; Węglarska } \\
\text { 1965; Durante Pasa \& Maucci } \\
\text { 1979; Dastych 1985; Klekowski \& } \\
\text { Opalinski 1986, 1989; De Smet et al. } \\
\text { 1988; Janiec 1996; Van Rompu \& De } \\
\text { Smet 1988, } 1996\end{array}$ \\
\hline & Platicrista angustata & (Murray, 1905) & $\begin{array}{l}\text { Sv, Fi, Tr, No, } \\
\text { NT, Op }\end{array}$ & $\begin{array}{l}\text { Murray 1907, Richters 19911b; } \\
\text { Marcus 1936; Durante Pasa \& } \\
\text { Maucci 1979; Dastych 1985; Maucci } \\
\text { 1996; Kaczmarek et al. 2012; } \\
\text { Zawierucha et al. 2013, 2015c, } 2017\end{array}$ \\
\hline & Platicrista itaquasconoide & $\begin{array}{l}\text { (Durante Pasa \& } \\
\text { Maucci, 1975) }\end{array}$ & ST & $\begin{array}{l}\text { *Meier, Mølnbukt (Agdenes, Sør- } \\
\text { Trøndelag) }\end{array}$ \\
\hline \multicolumn{5}{|c|}{ Pilatobiinae } \\
\hline & Pilatobius bullatus & (Murray, 1905) & ST & $\begin{array}{l}\text { *Meier, Søndre Knutshø (Oppdal, } \\
\text { Sør-Trøndelag) }\end{array}$ \\
\hline & $\begin{array}{l}\text { Pilatobius oculatus } \\
\text { oculatus }\end{array}$ & (Murray, 1906) & Op, Øf & $\begin{array}{l}\text { Richters 1911b; Durante Pasa \& } \\
\text { Maucci 1979; Dastych } 1985\end{array}$ \\
\hline & Pilatobius recamieri & (Richters, 1911) & Sv, Ho & $\begin{array}{l}\text { Richters 1911b; Marcus 1936; } \\
\text { Węglarska 1965; Dastych 1985; De } \\
\text { Smet \& Van Rompu 1994; Janiec } \\
\text { 1996; Maucci 1996; Meier 1996; Van } \\
\text { Rompu \& De Smet 1996; Kaczmarek } \\
\text { et al. 2012; Zawierucha et al. 2013, } \\
\text { 2015b,c, 2016a,b,d; Vonnahme et } \\
\text { al. 2016 }\end{array}$ \\
\hline \multicolumn{5}{|c|}{ Incerta subfamilia } \\
\hline & Mixibius saracenus & (Pilato, 1973) & ST & $\begin{array}{l}\text { *Meier, Søndre Knutshø (Oppdal, } \\
\text { Sør-Trøndelag) }\end{array}$ \\
\hline \multicolumn{5}{|c|}{ Microhypsibiidae } \\
\hline & Fractonotus caelatus & (Marcus, 1928) & ST & $\begin{array}{l}\text { *Meier, Søndre Knutshø (Oppdal, } \\
\text { Sør-Trøndelag) }\end{array}$ \\
\hline & Microhypsibius bertolanii & Kristensen, 1982 & $\mathrm{~Sv}$ & Zawierucha et al. 2016b \\
\hline & Microhypsibius truncatus & Thulin, 1928 & No, ST & $\begin{array}{l}\text { Durante Pasa \& Maucci 1979; Meier } \\
1996\end{array}$ \\
\hline \multicolumn{5}{|c|}{ Ramazzottiidae } \\
\hline & Hebesuncus conjungens & (Thulin, 1911) & Sv, Tr, No & $\begin{array}{l}\text { Marcus 1936; Durante Pasa \& } \\
\text { Maucci 1979; Dastych 1985, 2011; } \\
\text { Van Rompu \& De Smet 1991; } \\
\text { Kaczmarek et al. 2012; Zawierucha } \\
\text { et al. 2015c, 2016b }\end{array}$ \\
\hline & Ramazzottius cataphractus & (Maucci, 1974) & $\mathrm{Sv}$ & Dastych 1985 \\
\hline & Ramazzottius montivagus & (Dastych, 1983) & $\mathrm{Sv}$ & Dastych 1983, 1985 \\
\hline & Ramazzottius oberhaeuseri & (Doyère, 1840) & Sv, NT & $\begin{array}{l}\text { Richters 1903, 1904b, 1911a,b; } \\
\text { Murray 1907; Marcus 1936; } \\
\text { Węglarska 1965; Dastych } 1985\end{array}$ \\
\hline & Ramazzottius cf. rupeus & Biserov, 1999 & $\mathrm{~Sv}$ & Zawierucha et al. 2016b \\
\hline & Ramazzottius subanomalus & (Biserov, 1985) & Oslo & *Meier, Prinsdal (Oslo) \\
\hline
\end{tabular}


Table 2. Continued.

\begin{tabular}{|c|c|c|c|c|}
\hline Taxon & Species & Authority & County & Reference \\
\hline \multicolumn{5}{|c|}{ Isohypsibiodea } \\
\hline \multicolumn{5}{|c|}{ Isohypsibiidae } \\
\hline & Doryphoribius flavus & (Iharos, 1966) & ST & $\begin{array}{l}\text { *Meier, Søndre Knutshø (Oppdal, } \\
\text { Sør-Trøndelag) }\end{array}$ \\
\hline & Doryphoribius macrodon & Binda et al., 1980 & Sv, ST & $\begin{array}{l}\text { Binda et al. 1980; Dastych 1985; } \\
\text { Meier } 1996\end{array}$ \\
\hline & Eremobiotus alicatai & (Binda, 1969) & SF & $\begin{array}{l}\text { *Meier, Refvikstranda (Måløy, } \\
\text { Sogn og Fjordane) }\end{array}$ \\
\hline & Halobiotus arcturulius & $\begin{array}{l}\text { Crisp \& } \\
\text { Kristensen, } 1983\end{array}$ & $\mathrm{~Sv}$ & Mokievsky 1992 \\
\hline & Halobiotus crispae & Kristensen, 1982 & $\mathrm{~Sv}$ & $\begin{array}{l}\text { Møbjerg et al. 2007; Smykla et al. } \\
2011\end{array}$ \\
\hline & Halobiotus stenostomus & (Richters, 1908) & Tr, Ho & Richters 1908; Geddes 1968 \\
\hline & $\begin{array}{l}\text { Isohypsibius annulatus } \\
\text { annulatus }\end{array}$ & (Murray, 1905) & Sv, ST & $\begin{array}{l}\text { Murray 1907; Marcus 1936; Meier } \\
1996\end{array}$ \\
\hline & Isohypsibius asper & (Murray, 1906) & Sv, ST & $\begin{array}{l}\text { Richters 1903, 1911a; Murray 1907; } \\
\text { Meier 1996; Węglarska 1965; Łagisz } \\
1999 \text { (uncertain identification } \\
\text { according to the author) }\end{array}$ \\
\hline & Isohypsibius ceciliae & $\begin{array}{l}\text { Pilato \& Binda, } \\
1987\end{array}$ & Sv & Pilato \& Binda 1987 \\
\hline & Isohypsibius coulsoni & $\begin{array}{l}\text { Kaczmarek et al., } \\
2012\end{array}$ & $\mathrm{~Sv}$ & $\begin{array}{l}\text { Kaczmarek et al. 2012; Zawierucha } \\
\text { et al. 2013, 2015c, 2016b }\end{array}$ \\
\hline & Isohypsibius dastychi & Pilato et al., 1982 & $\mathrm{~Sv}$ & Pilato et al. 1982; Dastych 1985 \\
\hline & Isohypsibius elegans & $\begin{array}{l}\text { Binda \& Pilato, } \\
1971\end{array}$ & $\mathrm{~Sv}$ & $\begin{array}{l}\text { Dastych 1985; Van Rompu \& De } \\
\text { Smet 1996; Zawierucha et al. 2016b }\end{array}$ \\
\hline & $\begin{array}{l}\text { Isohypsibius granulifer } \\
\text { granulifer }\end{array}$ & Thulin, 1928 & Sv & $\begin{array}{l}\text { Dastych 1985; De Smet et al. 1988; } \\
\text { Van Rompu \& De Smet 1988, 1991, } \\
\text { 1996; De Smet \& Van Rompu 1994; } \\
\text { Janiec 1996; Zawierucha et al. 2015b }\end{array}$ \\
\hline & Isohypsibius karenae & Zawierucha, 2013 & Sv & Zawierucha 2013, 2016b \\
\hline & Isohypsibius marcellinoi & $\begin{array}{l}\text { Binda \& Pilato, } \\
1971\end{array}$ & $\mathrm{~Sv}, \mathrm{Fi}$ & $\begin{array}{l}\text { Meier 1996; Łagisz 1999; } \\
\text { Zawierucha et al. } 2013\end{array}$ \\
\hline & Isohypsibius marii & R. Bertolani, 1982 & ST & $\begin{array}{l}\text { *Meier, Agdenes fyr (Agdenes, Sør- } \\
\text { Trøndelag) }\end{array}$ \\
\hline & Isohypsibius monoicus & R. Bertolani, 1982 & $\mathrm{He}$ & Meier 1996 \\
\hline & $\begin{array}{l}\text { Isohypsibius papillifer } \\
\text { bulbosus }\end{array}$ & (Marcus, 1928) & $\mathrm{Sv}$ & Van Rompu \& De Smet 1988, 1991 \\
\hline & $\begin{array}{l}\text { Isohypsibius papillifer } \\
\text { papillifer }\end{array}$ & (Murray, 1905) & $\mathrm{Sv}$ & $\begin{array}{l}\text { Richters 1911b; Van Rompu \& De } \\
\text { Smet 1991, } 1996\end{array}$ \\
\hline & Isohypsibius pilatoi & $\begin{array}{l}\text { (Durante Pasa \& } \\
\text { Maucci, 1979) }\end{array}$ & $\mathrm{Fi}$ & Durante Pasa \& Maucci 1979 \\
\hline & $\begin{array}{l}\text { Isohypsibius prosostomus } \\
\text { prosostomus }\end{array}$ & Thulin, 1928 & $\begin{array}{l}\mathrm{Sv}, \mathrm{Fi}, \mathrm{Tr}, \mathrm{No}, \\
\mathrm{NT}, \mathrm{Bu}\end{array}$ & $\begin{array}{l}\text { Węglarska 1965; Mihelčič 1971; } \\
\text { Durante Pasa \& Maucci 1979; } \\
\text { Dastych 1985; Maucci 1996; Janiec } \\
\text { 1996; Zawierucha et al. 2015c }\end{array}$ \\
\hline & Isohypsibius pulcher & $\begin{array}{l}\text { (Mihelčič, } \\
\text { 1971/1972) }\end{array}$ & $\mathrm{Fi}$ & Mihelčič 1971, 1971/1972 \\
\hline & Isohypsibius reticulatus & Pilato, 1973 & Sv, ST & $\begin{array}{l}\text { Meier 1966; Zawierucha et al. } \\
\text { 2016b: cf. reticulatus }\end{array}$ \\
\hline & Isohypsibius sattleri & (Richters, 1902) & Sv, ST & $\begin{array}{l}\text { Dastych 1985; Meier 1996; } \\
\text { Zawierucha et al. 2015c, 2016b }\end{array}$ \\
\hline
\end{tabular}


Table 2. Continued.

\begin{tabular}{|c|c|c|c|c|}
\hline Taxon & Species & Authority & County & Reference \\
\hline & Isohypsibius schaudinni & (Richters, 1909) & $\mathrm{Sv}$ & Richters 1909; Węglarska 1965 \\
\hline & Isohypsibius tuberculatus & (Plate, 1888) & $\mathrm{Sv}$ & $\begin{array}{l}\text { Scourfield 1897; Schaudinn 1901; } \\
\text { Richters 1903; Murray 1907; Marcus } \\
\text { 1936; Zawierucha et al. 2016b }\end{array}$ \\
\hline & Thulinius augusti & (Murray, 1907) & $\begin{array}{l}\text { Sv, Fi, Tr, No, } \\
\text { NT, SF, Ho, } \\
\text { Ro, AA, VA, } \\
\text { Te, Bu, Ak }\end{array}$ & $\begin{array}{l}\text { Mihelčič 1971, Meier } 1996 \text { (both } \\
\text { records are uncertain identifications) }\end{array}$ \\
\hline
\end{tabular}

Macrobiotidae

Macrobiotus ariekammensis

Macrobiotus crenulatus

Macrobiotus echinogenitus

Macrobiotus hibiscus

Macrobiotus hufelandi

hufelandi
Węglarska, $1965 \quad$ Sv

Richters, 1904

Richters, 1904

de Barros, 1942

C.A.S. Schultze, 1834

Bertolani et al. 1993

Macrobiotus norvegicus

Macrobiotus occidentalis

occidentalis

Macrobiotus pallarii

Macrobiotus persimilis

Macrobiotus polonicus

Macrobiotus recens

Macrobiotus spectabilis

Macrobiotus vladimiri
Mihelčič, 1971/1972

Murray, 1910

Maucci, 1954

Binda \& Pilato, 1972

Pilato et al., 2003

Cuénot, 1932

Thulin, 1928

Bertolani et al. 2011
Węglarska 1965; Dastych 1985; Łagisz 1999

Richters 1904b; Murray 1907; Maucci 1996; Van Rompu \& De Smet 1996; Kaczmarek et al. 2012; Zawierucha 2013; Zawierucha et al. 2013, 2015c, 2016b, 2017

Sv, Fi, Tr, No, Richters 1903, 1904b, 1911a,b; $\mathrm{Op}$ Murray 1907; Węglarska 1965; Durante Pasa \& Maucci 1979; Dastych 1985; Klekowski \& Opalinski 1986, 1989; De Smet et al. 1988; Van Rompu \& De Smet 1991, 1996; Meier 1996; Zawierucha et al. 2013

Durante Pasa \& Maucci 1979

Sv, Fi, Tr, No, Scourfield 1897; Schaudinn 1901; NT, ST, Op, Richters 1903, 1904a,b, 1911b; $\mathrm{Bu}, \mathrm{Ho}$ Murray 1907; Summerhayes \& Elton 1923; Marcus 1936; Węglarska 1965; Durante Pasa \& Maucci 1975, 1979; Dastych 1985; Van Rompu \& De Smet 1988; Meier 1996; Kaczmarek et al. 2012; Zawierucha et al. 2013, 2015c

*Meier, Hafjelltoppen (Øyer, Oppland)

Mihelčič 1971, 1971/1972

Węglarska 1965; Durante Pasa \& Maucci 1979

Durante Pasa \& Maucci 1979

*Meier, Søndre Knutshø (Oppdal, Sør-Trøndelag)

*Meier, Havna (Tjøme, Vestfold)

* Meier, Austråt (Ørland, SørTrøndelag)

Durante Pasa \& Maucci 1979; Klekowski \& Opaliński 1986, 1989

*Meier \& Ekrem, Kalvskinnet (Trondheim, Sør-Trønderlag) 
Table 2. Continued.

\begin{tabular}{|c|c|c|c|c|}
\hline Taxon & Species & Authority & County & Reference \\
\hline & $\begin{array}{l}\text { Mesobiotus harmsworthi } \\
\text { harmsworthi }\end{array}$ & (Murray, 1907) & $\begin{array}{l}\text { Sv, Fi, No, } \\
\text { Op }\end{array}$ & $\begin{array}{l}\text { Murray 1907; Richters 1911a,b; } \\
\text { Węglarska 1965; Durante Pasa \& } \\
\text { Maucci 1975, 1979; Dastych 1985; } \\
\text { Klekowski \& Opalinski 1986, 1989; } \\
\text { Maucci 1996; Meier 1996; Van } \\
\text { Rompu \& De Smet 1996; Lagisz } \\
\text { 1999; Kaczmarek et al. 2012; } \\
\text { Zawierucha et al. 2013, 2015c, } \\
\text { 2016b, } 2017\end{array}$ \\
\hline & $\begin{array}{l}\text { Mesobiotus harmsworthi } \\
\text { obscurus }\end{array}$ & (Dastych, 1985) & $\mathrm{Sv}$ & $\begin{array}{l}\text { Dastych 1985; Maucci 1996; } \\
\text { Kaczmarek et al. 2012; Zawierucha } \\
\text { et al. 2015c, 2016b }\end{array}$ \\
\hline & Mesobiotus montanus & (Murray, 1910) & Fi, NT, Op & Durante Pasa \& Maucci 1979 \\
\hline & Mesobiotus nuragicus & $\begin{array}{l}\text { (Pilato \& } \\
\text { Sperlinga, 1975) }\end{array}$ & Но & $\begin{array}{l}\text { *Meier, Bondhusvannet (Kvinn- } \\
\text { herad, Hordaland) }\end{array}$ \\
\hline & Mesobiotus orcadensis & (Murray, 1907) & $\mathrm{Fi}, \mathrm{Tr}$, No & Durante Pasa \& Maucci 1975, 1979 \\
\hline & Mesobiotus peterseni & (Maucci, 1991) & $\mathrm{Sv}$ & Jankovská et al. 2016 \\
\hline & Minibiotus crassidens & (Murray, 1907) & $\mathrm{Sv}$ & Węglarska 1965 \\
\hline & Minibiotus cf. formosus & $\begin{array}{l}\text { Zawierucha et al., } \\
2014\end{array}$ & $\mathrm{~Sv}$ & Zawierucha et al. 2016b \\
\hline & Minibiotus harrylewisi & $\begin{array}{l}\text { Meyer \& Hinton, } \\
2009\end{array}$ & Oslo & *Meier, Hvervenbukta (Oslo) \\
\hline & Minibiotus intermedius & (Plate, 1888) & $\begin{array}{l}\text { Sv, Tr, No, } \\
\text { NT, Op, He, } \\
\text { Øf, Ho }\end{array}$ & $\begin{array}{l}\text { Richters 1903, 1904a,b, 1911b; } \\
\text { Murray 1907; Marcus 1936; Durante } \\
\text { Pasa \& Maucci 1975, 1979; Lagisz } \\
\text { 1999; Jankovská et al. 2016: cf. } \\
\text { intermedius }\end{array}$ \\
\hline & Paramacrobiotus areolatus & (Murray, 1907) & Sv, ST & $\begin{array}{l}\text { Murray 1907; Richters 1911b; } \\
\text { Marcus 1936; Węglarska 1965; } \\
\text { Dastych 1985; Maucci 1996; Meier } \\
\text { 1996; Lagisz 1999; Zawierucha et } \\
\text { al. 2015c }\end{array}$ \\
\hline
\end{tabular}

Paramacrobiotus richtersi （Murray, 1911)

Tenuibiotus voronkovi

Tenuibiotus willardi

Murrayidae
Dactylobiotus ambiguus

Dactylobiotus ampullaceus

Dactylobiotus dispar
(Murray, 1907)

(Thulin, 1911)

(Murray, 1907)
Sv, Tr, NT, Węglarska 1965; Mihelčič 1971; ST, Op, Øf Durante Pasa \& Maucci 1979; Dastych 1985; Lagisz 1999; Zawierucha et al. 2016b

Sv

Sv

Tumanov 2007; Zawierucha et al. 2013, 2016b

Dastych 1985 (see Zawierucha et al. 2016c); Maucci 1996

Sv, Fi, ST, Op Murray 1907; Mihelčič 1971; De Smet et al. 1988; Van Rompu \& De Smet 1988; Janiec 1996; Meier 1996; Maucci 1996

ST

*Meier, Søndre Knutshø (Oppdal, Sør-Trøndelag)

Sv，ST，Op， Murray 1907; Marcus 1936; Mihelčič Ak, Bu, SF 1971; Klekowski \& Opalinski 1986, 1989; Van Rompu \& De Smet 1991, 1996; Meier 1996 
Table 2. Continued.

\begin{tabular}{|c|c|c|c|c|}
\hline Taxon & Species & Authority & County & Reference \\
\hline & Dactylobiotus macronyx & (Dujardin, 1851) & $\mathrm{Sv}$ & $\begin{array}{l}\text { Richard 1898; Schaudinn 1901; } \\
\text { Richters 1903; Summerhayes \& } \\
\text { Elton } 1923\end{array}$ \\
\hline & Dactylobiotus octavi & $\begin{array}{l}\text { Guidetti et al., } \\
2006\end{array}$ & Op & $\begin{array}{l}\text { *Meier, Hafjelltoppen (Øyer, } \\
\text { Oppland) }\end{array}$ \\
\hline & Murrayon dianeae & (Kristensen, 1982) & $\mathrm{Sv}$ & Van Rompu \& De Smet 1991, 1996 \\
\hline & Murrayon hastatus & (Murray, 1907) & $\mathrm{ST}, \mathrm{Bu}, \mathrm{Vf}$ & $\begin{array}{l}\text { *Meier, Søndre Knutshø (Oppdal, } \\
\text { Sør-Trøndelag) }\end{array}$ \\
\hline & Murrayon hibernicus & (Murray, 1911) & Sv, No, NT & $\begin{array}{l}\text { Durante Pasa \& Maucci 1979; } \\
\text { Dastych 1985; Lagisz 1999; } \\
\text { Zawierucha et al. } 2013\end{array}$ \\
\hline & Murrayon pullari & (Murray, 1907) & Sv, Fi, ST, Op & $\begin{array}{l}\text { Murray 1907; Marcus 1936; De } \\
\text { Smet et al. 1987, 1988; Meier } 1996\end{array}$ \\
\hline \multicolumn{5}{|c|}{ Richtersiidae } \\
\hline & Adorybiotus granulatus & (Richters, 1903) & Tr, NT, ST & $\begin{array}{l}\text { Richters 1903, 1904b; Durante Pasa } \\
\text { \& Maucci 1975, 1979; Meier } 1996\end{array}$ \\
\hline & $\begin{array}{l}\text { Diaforobiotus islandicus } \\
\text { islandicus }\end{array}$ & (Richters, 1904) & Sv, Fi, Tr, No & $\begin{array}{l}\text { Murray 1907; Węglarska 1965; } \\
\text { Durante Pasa \& Maucci 1975, } \\
\text { 1979; Dastych 1985; Klekowski } \\
\text { \& Opalinski 1986, 1989; Maucci } \\
\text { 1996; Łagisz 1999; Dastych 2011: cf. } \\
\text { islandicus; Kaczmarek et al. 2012; } \\
\text { Jankovská et al. 2016; Zawierucha et } \\
\text { al. 2013, 2015a,c, 2016b }\end{array}$ \\
\hline & Richtersius coronifer & (Richters, 1903) & $\begin{array}{l}\text { Sv, Tr, No, } \\
\text { NT }\end{array}$ & $\begin{array}{l}\text { Richters 1903, 1904b; Węglarska } \\
\text { 1965; Durante Pasa \& Maucci 1975, } \\
\text { 1979; Dastych 1985; Maucci 1996; } \\
\text { Łagisz 1999; Zawierucha et al. } \\
\text { 2015c, 2016b }\end{array}$ \\
\hline
\end{tabular}

species» (Degma et al. 2017), although some are questionable, probable synonyms or should be split into separate species (see comments to the checklist below). 121 species are retrieved from published literature and another 25 new species to Norway (Table 3) based on own findings over many years.

Samples of mosses, lichens and detritius from ponds and streams were collected and kept in a freezer at $-18^{\circ} \mathrm{C}$. Prior to examination the samples were soaked in water for two hours, then shaked in a beaker for two minutes, and finally the detritus from the bottom of the beaker was decanted over a Petri dish. Hoyer was used for preparation of permanent slides. Mounted slides were kept on a hot plate for one week at $55^{\circ} \mathrm{C}$. A Zeiss Axiostar plus light microscope, with oil immersion on the objective (x100) and the condenser was used for identification of the species. Slides with these species are currently in the author's collection, but will be transferred to the invertebrate collection at the NTNU University Museum in Trondheim together with a reference collection of Norwegian tardigrades.

\section{Comments to the checklist}

For a long time the genus Milnesium was considered monotypic with Milnesium tardigradum Doyère, 1840 as the only, cosmopolitan species (Tumanov 2006). In recent years new taxonomic criteria have revealed a greater variety in morphology (Pilato 1981; Maucci 1988 (1991); Binda \& Pilato 1990) and over some years more than 30 new species have been added to the genus (see: Michalczyk et al. 2012a,b; Degma et al. 2016; Morek et al. 2016). Nine Norwegian studies have reported Milnesium tardigradum Doyère, 1840, (Richters 1903, 1904b, 1911a,b; Węglarska 1965; Durante Pasa \& Maucci 1975, 1979; Maucci 1996; Dastych 2011) some of them published before the most recent taxonomic revisions. It is therefore not unlikely that re-examination of these specimens and/or new material in light of new taxonomic criteria will uncover a greater diversity of Milnesium species from Norway.

Macrobiotus hufelandi Schultze, 1834 was the first tardigrade described and for many years recorded in faunistic studies from many localities worldwide and therefore considered cosmopolitan (Ramazzotti \& Maucci, 1983). To make valid identification of species in the hufelandi-group the following characters from the taxonomic toolbox must be taken into consideration; $p t$ values, granulation and pores in the cuticula, dentate or smooth lunula and egg chorion structures. Many of these characters are not mentioned in numerous studies from 


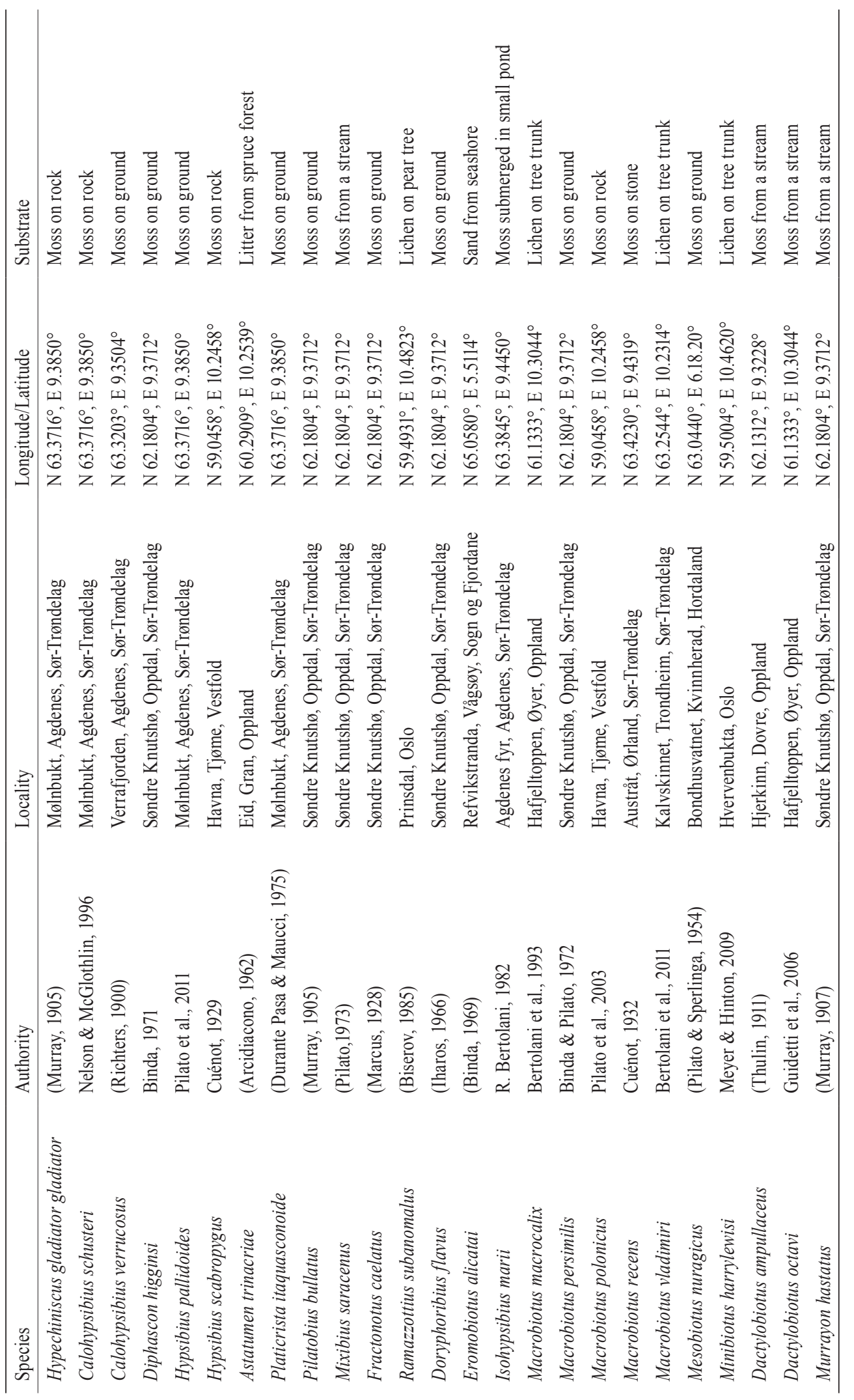




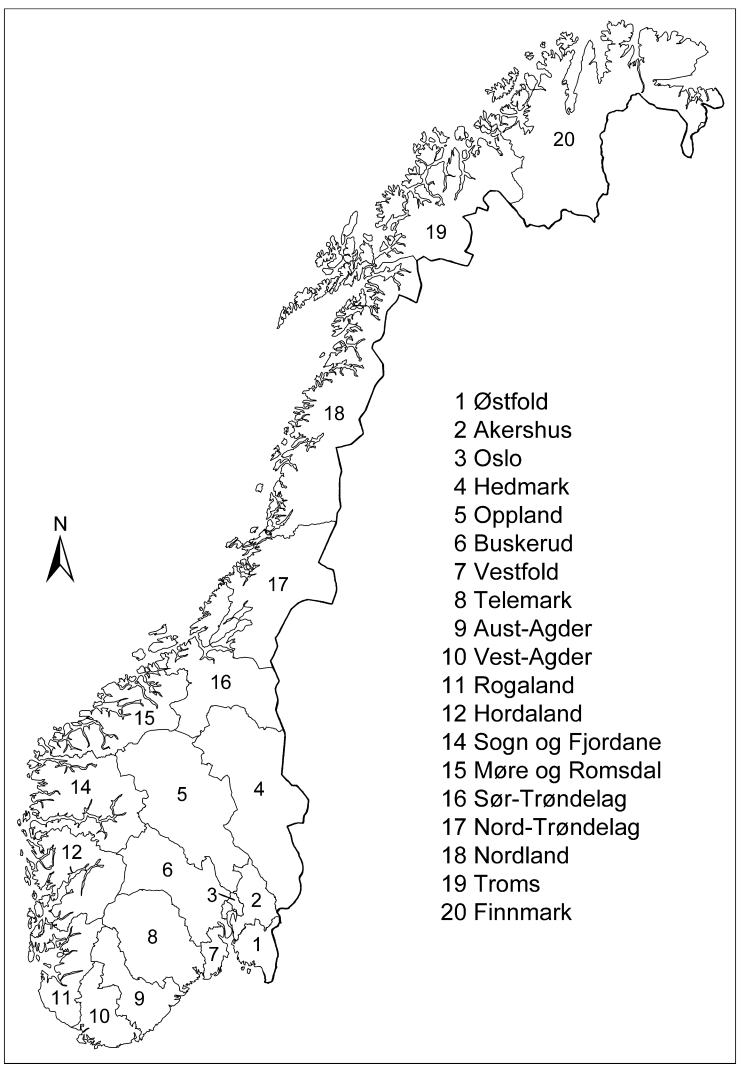

Figure I. Counties in Norway per December 2017 numbered according to Statistics Norway. Map source: www.geonorge.no.

Table 4. Number of species found in different Norwegian counties.

\begin{tabular}{lccc}
\hline & County & & \\
\cline { 1 - 3 } Name & Numbers in & Abbrev. in & Number \\
Table 2 & of species \\
\hline Svalbard & Figure 1 & Sv & 97 \\
Nordland & 18 & No & 37 \\
Sør-Trøndelag & 16 & ST & 37 \\
Oppland & 5 & Op & 32 \\
Troms & 19 & Tr & 30 \\
Finnmark & 20 & Fi & 26 \\
Nord-Trøndelag & 17 & NT & 21 \\
Buskerud & 6 & Bu & 8 \\
Hordaland & 12 & Ho & 8 \\
Østfold & 1 & Øf & 7 \\
Sogn og Fjordane & 14 & SF & 3 \\
Vestfold & 7 & Vf & 3 \\
Hedmark & 4 & He & 3 \\
Oslo & 3 & Oslo & 2 \\
Akershus & 2 & Ak & 2 \\
Vest-Agder & 10 & VA & 2 \\
Aust-Agder & 9 & AA & 2 \\
Telemark & 8 & Te & 1 \\
Rogaland & 11 & Ro & 1 \\
Møre og Romsdal & 15 & - & 0 \\
\hline
\end{tabular}

the early $20^{\text {th }}$ century. Thirteen publications report to have found Macrobiotus hufelandi from Norway, but most of them do not present any detailed discussion of important taxonomic characters. Thus, correct species attribution is questionable.

Durante Pasa \& Maucci (1979) recorded Acutuncus antarcticus (Richters, 1904) from Bodø, but it is not listed in the current checklist since his identification is dubious. A. antarcticus was redescribed by Dastych (1991) who discussed the possibility of synonymy between $A$. antarcticus and Hypsibius arcticus (Murray, 1907). The taxonomic status of $H$. arcticus is unsettled because of its inadequate description (Richters, 1904b) and lack of type material. Acuntuncus antarcticus was described from Antarctica and Hypsibius arcticus from the Arctic and only a redescription of the latter taxon from the type locality can contribute to a solution of this question.

Mihelčič (1971/1972) described three limnic species from Norway; Macrobiotus norvegicus from Kuvatn, Finnmark, Itaquascon enckelli from Sagelva, Troms (and also reported it from localities in Sweden and Finland) and Isohypsibius pulcher from Lakselv, Finnmark (also reported from Denmark and Sweden). Unfortunately his descriptions lack photos and his poor drawings emphasize the fact that these three species require redescription. In the description of $M$. norvegicus Mihelčič wrote: «Die glatten Eier werden in die alte Kutikula abgelegt». All known species of the superfamily Macrobiotoidea (except Dactylobiotus macronyx) lay their ornamented eggs freely. Therefore his identification is dubious and the type locality should be revisited in order to solve the true systematic position of this species.

Schaudinn (1901) found five specimens of Calohypsibius ornatus var. spinosissimus from Bjørnøya; Dastych (1985) reported C. ornatus var. carpaticus from Svalbard and Durante Pasa and Maucci (1979) found C. ornatus y-septemcinctus from Norway. They have not been included in the checklist due to the fact that their taxonomic status is doubtful. The morphological variation within the species was thoroughly studied by Bartoš (1940) and he reported as many as 32 different forms. He divided these varieties in two separate groups; Calohypsibius ornatus typicus $(1-13)$ and Calohypsibius ornaus carpaticus (14 - 32), without giving a name to each variety. It seems likely that all these forms should not be treated as valid denominations, but considered as natural intraspecific variability (Durante Pasa and Maucci, 1979). Calohypsibius ornatus occur quite often in Hylocomnium sp. mosses in Norway and the great variation described by Bartoš has not been observed. However, the dispute regarding the status of the Calohypsibius ornatus species complex is not brought to an end. For instance Pilato (1989) states that «under the name Calohypsibius ornatus various species, and perhaps genera, very different from each other as regards the characters of the bucco-pharyngeal apparatus are gathered.» 


\section{DISCUSSION}

In total 146 species of Tardigrada are recorded from Norwegian territory (Table 2); whereof 102 species recorded from the mainland and 97 species from Svalbard. From neighbouring Sweden 101 species are found (Guidetti et al. 2015). 74 species are found in both countries - that gives a total of 172 species recorded from the Scandinavian peninsula and Svalbard. 43 species are recorded from Svalbard and not found in mainland Norway. There are no investigations that support an assumption that these species are found on Svalbard only, and not in similar climate and biotopes in mountainous mainland Norway. Therefore there is reason to assume that the number of species from both Sweden and Norway are underestimated.

Meier (1996) recorded 37 species from limnic biotopes like streams and ponds. Species from the genera Pseudechiniscus, Echiniscus, Macrobiotus, Paramacrobiotus, Diphascon, Astatumen and Adropion sometimes occur submerged in water. Occasionally, by heavy rainfalls, terrestrial species from the genera mentioned above are wasted into water environments and therefore the real status of these species as genuine limnic species are questionable.

Our knowledge of Norwegian marine tardigrades is almost non-existent. Only seven species have been reported so far (Richters 1908; Tambs-Lyche, 1940; Geddes 1968; Mokievsky 1992; Møbjerg et al., 2007; Kristensen \& Hallas 2008; Ringvold 2015; Smykla et al., 2011) and due to the great variation of biotopes along the Norwegian coastline there is reason to believe that the real number of species is considerably higher. For instance, Hansen et al. (2001) reported 35 different species of Heterotardigrada from the Faroe Bank, which emphasize the great potential of investigating tardigrade species from the marine environment along the Norwegian coast and offshore banks.

Although 146 species are recorded from Norway so far, there is no doubt that most of the country lack thorough knowledge of tardigrade dispersal and number of species (Table 4). The Tardigrada fauna of Svalbard is best documented with 97 different species recorded. Records of Tardigrada from western and southern part of the country are scarce or hardly mentioned in literature. The number of species found in each county reflect the road followed by Mihelčič $(1971,1971 / 1972)$ and Durante Pasa and Maucci $(1975,1979)$ when they collected moss, lichens and samples from freshwater during their travel in Norway. They both started collecting in eastern Finnmark following the main road towards south and ending up in Østfold. Consequently the greatest number of species are found in the counties along their travelling route. Best known is the fauna of Nordland and Sør-Trøndelag with 37 species each, while Møre og Romsdal remain without any records of Tardigrada.

\section{ACKNOWLEDGMENTS}

I am very grateful to Professor Torbjørn Ekrem for valuable comments to the manuscript and checklist.

\section{REFERENCES}

Bartoš E. 1940. Über die variation der art Hypsibius ornatus Richt. (Tardigrada). Zoologische Jahrbücher, Abteilung für Systematik 73: 369-384.

Bertolani R. 1987. Sexuality, reproduction and propagation in tardigrades. In R. Bertolani (ed.), Biology of Tardigrades. Proceedings of the 4th International Symposium on the Tardigrada. September 1985, Modena, Italy. Selected Symposia and Monographs 1: 93-101. Mucchi, Modena, Italy.

Bertolani R, Rebecchi L. 1993. A revision of the Macrobiotus hufelandi group (Tardigrada, Macrobiotidae), with some observations on the taxonomic characters of eutardigrades. Zoologica Scripta 22: 127-152. doi: 10.1111/j.1463-6409.1993. tb00347.x

Binda MG, Pilato G. 1990. Tardigradi del Terra del Fuoco e Magallanes. I. Milnesium brachyungue, nuova specie di Tardigrado Milnesiidae. Animalia 17: 105-110.

Binda MG, Pilato G, Dastych H. 1980. Descrizione di una nuova species di Eutardigrado: Doryphoribius macrodon. Animalia 7: 23-27.

Clausen C. 2004. A new species of Acanthodasys (Gastrotricha: Thaumastodermatidae) from the west coast of Norway. Sarsia 89: 137-141. doi: 10.1080/00364820410004972

Coulson SJ, Convey P, Aakra K, Aarvik L, Ávila-Jiménez ML, Babenko A, Biersma E, Boström S, Brittain JE, Carlsson A, Christoffersen K, De Smet WH, Ekrem T, Fjellberg A, Füreder L, Gustafsson D, Gwiazdowicz DJ, Hansen LO, Hullé M, Kaczmarek Ł, Kolicka M, Kuklin V, Lakka HK, Lebedeva N, Makarova O, Maraldo K, Melekhina E, Ødegaard F, Pilskog HE, Simon JC, Sohlenius B, Solhøy T, Søli G, Stur E, Tanasevich A, Taskaeva A, Velle G, Zawierucha K, Zmudczyńska-Skarbek K. 2014. The terrestrial and freshwater invertebrate biodiversity of the archipelagos of the Barents Sea, Svalbard, Franz Josef Land and Novaya Zemlya. Soil Biology and Biochemestry 68: 440-470. doi: 10.1016/j.soilbio.2013.10.006

Dastych H. 1983. Two new Eutardigrada species from West Spitsbergen and the Tatra Mts. Bulletin de la Societe des Amis des Sciences et des Lettres de Poznan, Serie D, sciences biologiques 23: 195-200.

Dastych H. 1985. West Spitsbergen Tardigrada. Acta Zoologica Cracoviensia 28: 169-214.

Dastych H. 1987. Two new species of Tardigrada from the Canadian Subartic with some notes on dimorphism in the family Echiniscidae. Mitteilungen Hamburgisches Zoologisches Museum 8: 319-334.

Dastych H. 1991. Redescription of Hypsibius antarcticus (Richters, 1904), with some notes on Hypsibius arcticus (Murray 1907) (Tardigrada). Mitteilungen aus dem Hamburgischen Zoologischen Museum und Institut 88: 141-159.

Dastych, H. 2011. Bergtrollus dzimbowski gen. n., sp. n., a remarkable new tardigrade genus and species from the nival zone of the Lyngen Alps, Norway (Tardigrada: Milnesiidae). 
Entomologische Mitteilungen aus dem Zoologischen Museum Hamburg 15: 335-359.

Degma P, Guidetti R. 2007. Notes to the current checklist of Tardigrada. Zootaxa, 1579, 41-53.

Degma P, Bertolani R, Guidetti R. 2017. Actual checklist of Tardigrada species. 45 pp. Available from: http://www.tardigrada. modena.unimo.it/miscellanea/Actual $\% 20$ checklist $\% 20$ of $\% 20$ Tardigrada.pdf (Accessed 1st. of October 2017)

De Smet WH, Van Rompu EA. 1994. Rotifera and Tardigrada from some cryoconite holes on a Spitsbergen (Svalbard) glacier. Belgian Journal of Zoology 124: 27-37.

De Smet WH, Van Rompu EA, Beyens L. 1987. Rotifera, Gastrotricha en Tardigrada uit Shetland, de Faroër en Spitsbergen. Natuurwetten Tijdschrift 69: 81-102.

De Smet WH, Van Rompu EA, Beyens L. 1988. Contribution to the Rotifers and aquatic Tardigrada of Edgeøya (Svalbard). Fauna norvegica. Serie A 9: 19-30.

Durante Pasa MV, Maucci W. 1975. Descrizione di tre nuove specie di Tardigradi della Scandinavia. Atti Società Italiana di Scienze Naturali e del Museo Civico di Storia Naturale di Milano 116: 244-250.

Durante Pasa MV, Maucci W. 1979. Moss Tardigrada from the Scandinavian Peninsula. Second International Symposium on Tardigrada, Krakow, Poland, July 28 - 30 1977, Zeszyty Naukowe Uniwersytetu Jagiellonskiego, Prace Zoologiczne, Krakow 79: 47-85.

Geddes DC. 1968. A note on the marine tardigrade Hypsibius (Isohypsibius) stenostomus (Richters) from the Tromsø area, Northern Norway. Astarte 33: 1-4.

Goës AT. 1862. Om tardigrader, Anguillulae m.m. från Spetsbergen. Öfversigt af Kungliga Vetenskaps-akademiens Förhandlingar 1862: 18.

Goeze JAE. 1773. Über der Kleinen Wasserbär. Page 67 in: H. K. Bonnet, editor. Abhandlungen aus der Insectologie, Ubers. Usw, 2. Beobachtg.: 367 - 375.

Guidetti R, Altiero T, Rebecchi L. 2011. On dormancy strategies in tardigrades. Journal of Insect Physiology 57: 567-576 doi: 10.1016/j.jinsphys.2011.03.003

Guidetti R, Jönsson I, Møbjerg Kristensen R. 2015. Tardigrades of Sweden; an updated check-list. Zootaxa 3981 (4): 491-507. doi: 10.11646/zootaxa.3981.4.2

Hansen JG, Jørgensen A, Møbjerg Kristensen R. 2001. Preliminary studies of the Tardigrada fauna of the Faroe Bank. Zoologisher Anzeiger 240: 385-393. doi: 10.1078/0044-5231-00046

Janiec K. 1996. The comparison of freshwater invertebrates of Spitsbergen (Artic) and King George Island (Antartic). Polar Polish Research 17: 173-202.

Jankovská V, Roszkowska M, Kaczmarek Ł. 2016. Remains of non-pollen-palynomorphs-tardigrades from Spitsbergen found during pollen analyses. Polar Record 52: 450-463. doi: 10.1017/ S0032247416000127

Kaczmarek Ł, Zawierucha K, Smykla J, Michalczyk Ł. 2012. Tardigrada of the Revdalen (Spitsbergen) with the description of two new species: Bryodelphax parvuspolaris (Heterotardigrada) and Isohypsibius coulsoni (Eutardigrada). Polar Biology 35: 1013-1026. doi: 10.1007/s00300-011-1149-0

Klekowski RZ, Opalinski KW. 1986. Matter and energy flow in Spitsbergen ornithogenic tundra. Polar Research 4: 187-197.

Klekowski RZ, Opalinski KW. 1989. Oxygen consumption in
Tardigrada from Spitsbergen. Polar Biology 9: 299-303.

Kristensen RM, Hallas TE. 1980. The tidal genus Echiniscoides and its variability, with erection of Echiniscoididae fam. $n$. (Tardigrada). Zoologica Scripta 9: 113 - 127. doi: 10.1111/j.14636409.1980.tb00657.x

Łagisz M. 1999. Fauna niesporczaków (Tardigrada) na nunatakach Spitsbergenu jako przyklad fauny wyspowej. (The water bears (Tardigrada) from Spitsbergen nunataks as an example of insular fauna.) MSc. thesis, Jagiellonian University, Krakow.

Łokas E, Zaborska A, Kolicka M, Różycki M, Zawierucha K. 2016. Accumulation of atmospheric radionuclides and heavy metals in cryoconite holes on an Arctic glacier. Chemosphere 160: 162172. doi: 10.1016/j.chemosphere.2016.06.051

Marcus E. 1936. Das Tierreich. Tardigrada. Berlin und Leipzig 66: $1-340$.

Maucci W. 1996. Tardigrada of the Arctic tundra with description of two new species. Zoological Journal of the Linnean Society 116: 185-204. doi: 10.1111/j1096-3642.1996.tb02343.x

Maucci W. 1988 (1991). Tre nouve specie di Eutardigrai della Groenlandia Meridionale. Bolletino del Museo Civico di Storia Naturale di Verona 15: 279-289.

Meier T. 1996. Tardigrada, 89-91. In: Limnofauna Norvegica. Katalog over norsk ferkvannsfauna. (Aagaard K. \& Dolmen D., eds.), Tapir Forlag, Trondheim.

Michalczyk Ł, Welnicz W, Frohme M, Kaczmarek Ł. 2012a. Redescription of three Milnesium Doyère, taxa (Tardigrada: Eutardigrada: Milnesiidae), including the nominal species for the genus. Zootaxa 3154: 1-20.

Michalczyk Ł, Welnicz W, Frohme M, Kaczmarek Ł. 2012 b. Corrigenda of Zootaxa, 3154: 1 - 20. Redescription of three Milnesium Doyère, taxa (Tardigrada: Eutardigrada: Milnesiidae), including the nominal species for the genus. Zootaxa 3393: 66-68.

Mihelčič F. 1971. Süsswassertardigraden aus Nordeuropa. Entomologica Scandinavica 2: 205-214. doi: 10.1163/187631271X00211

Mihelčič F. 1971/1972. Ein Beitrag zur Kenntnis der Süsswassertardigraden Nordeuropas. Verhandlungen der Zoologisch-Botanischen Gesellschaft in Wien 110/111: 37-45.

Mokievsky VO. 1992. Composition and distribution of intertidal meiofauna of Isfjorden, West Spitsbergen. Polish Polar Research 13: $31-40$.

Morek W, Gąsiorek P, Stec D, Blagden B, Michalczyk Ł. 2016. Experimental taxonomy exposes ontogenetic variability and elucidates the taxonomic value of claw configuration in Milnesium Doyère, 1840 (Tardigrada: Eutardigrada: Apochela). Contribution to Zoology 85: 173-200.

Murray J. 1907. Arctic Tardigrada, collected by Wm. S. Bruce. Transactions of the Royal Society of Edinburgh 45: 669-681. doi: 10.1017/S0080456800011777

Møbjerg N, Jørgensen A, Eibye-Jacobsen J, Halberg KA, Persson D, Kristensen RM. 2007. New records on cyclomorphosis in the marine eutardigrade Halobiotus crispae (Eutardigrada: Hypsibiidae). Journal of Limnology 66: 132-140. doi: 10.4081/ jlimnol.2007.07.s1.132

Møbjerg N, Halberg KA, Jørgensen A, Persson D, Bjørn M, Ramløv H, Kristensen RM. 2011. Survival in extreme environments - on the current knowledge of adaptations in tardigrades. Acta Physiologica 202: 409-420. doi: 10.1111/j.1748-1716.2011.02252x Nelson DR. 2002. Current Status of the Tardigrada: Evolution and 
Ecology. Integrative and Comparative Biology 42: 652-659. doi: 10.1093/icb/42.3.652

Pilato G. 1981. Analisi di nuovi caratteri nello studio degli Eutardigradi. Animalia 8: 51-57.

Pilato G. 1989. Phylogenesis and systematic arrangement of the family Calohypsibiidae Pilato, 1969 (Eutardigrada). Zeitschrift für Zoologische Systematik und Evolutionsforschung 27: 8-13.

Pilato G, Bertolani R, Binda MG. 1982. Studio degli Isohypsibius del gruppo elegans (Eutardigrada, Hypsibiidae) con descrizione di due nuove specie. Animalia 9: 185-198.

Pilato G, Binda MG. 1987. Isohypsibius ceciliae, nuova specie di Eutardigrado (Hypsibiidae) dello Spitsbergen. Animalia 14: 125-129.

Pilato G, Sabella G, Lisi O. 2016. Two new species of Milnesium (Tardigrada: Milnesiidae). Zootaxa 4132: 575-587. doi: 10.11646/ zootaxa.4132.4.9

Rahm G. 1931. Tardigrada of the South of America. Revista Chilena de Historia Natural 35: 118-141.

Ramazzotti G, Maucci, W. 1983. Il Phylum Tardigrada. Memorie dell'istituto Italiano di Idrobiologia, 41, 1-1012.

Richard J. 1898. Sur la faune des eaux douces explorées en 1898 pendant la campagne du yacht Princesse-Alice (Lofoten, Spitsberg, Iles Beeren, Hope, de Barents et Færoer). Mémoires de la Sociét'zoologique de France 11: 326-338.

Richters F. 1903. Nordische Tardigraden. Zoologischer Anzeiger 27: 168 - 172.

Richters F. 1904a. Beitrag zur Verbreitungen der Tardigraden im südlichen Scandinavien und an der mecklenburgischen Küste. Zoologischer Anzeiger 28: 347-352.

Richters F. 1904b. Arktische Tardigraden. Fauna Arctica 3: 494508.

Richters F. 1908. Marine Tardigraden. Zoologischer Anzeiger 33: 77-85.

Richters F. 1909. Tardigraden-Studien. Berichte der Senckenbergischen Naturforschenden Gesellschaft in Frankfurt am Main 40: 28-45.

Richters F. 1911a. Moosfauna, Tardigrada. In A. Koenig. Avifauna Spitzbergensis 283-286.

Richters F. 1911b. Faune des mousses. Tardigrades. Duc d'Orleans. Campagne arctique de 1907. Imprimerie Scientifique C. Buelens, Bruxelles: 1-20, pl. I-III.

Ringvold H. 2015. Bjørnedyr i Norge - fra land, ferskvann og saltvann. Fauna 68: 70-75 (in Norwegian)

Schaudinn F. 1901. Die Tardigraden. Fauna Arctica, Jena 22: 186195.

Schultze CAS. 1834. Macrobiotus Hufelandii, animal $e$ crustaceorum classe novum, reviviscendi post diuturnam asphyxiam et ariditatem potens. Curths, Berlin, 6 pp., 1 table.

Scourfield DJ. 1897. Contributions to the Non-Marine Fauna of Spitsbergen. Part I. Preliminary notes, and report on the Rhizopoda, Tardigrada, Entomostraca, \& C. Proceedings of the Zoological Society of London 784-792.

Smykla J, Kaczmarek Ł, Huzarska K, Michalczyk Ł. 2011. The first record of a rare marine tardigrade, Halobiotus crispae Kristensen, 1982 (Eutradigrada; Hypsibiidae), from the Svalbard Archipelago. Polar Biology 34: 1243-1247. doi: 10.1007/s00300-011-0986-1

Sommerhayes VS, Elton CS. 1923. Contribution to the ecology of Spitsbergen and Bear Island. Journal of Ecology 11: 214-286.
Spallanzani L. 1776. Opuscoli di fisica animale, e vegetabile, Vol. 2, Il Tardigrado etc., Opusc. 4, sez, spec., pp. 181 - 253, Modena, Italy.

Tambs-Lyche H. 1940. Marine Tardigraden bei Bergen. Bergens Museums Årbok 1939 - 40. Naturvitenskapelig rekke Nr. 5: 1-5. Tumanov DV. 2006. Five new species of the genus Milnesium (Tardigrada, Eutardigrada, Milnesiidae). Zootaxa 1122: 1-23.

Tumanov DV. 2007. Three new species of Macrobiotus (Eutardigrada, Macrobiotidae, tenuis-group) from Tien Shan (Kirghizia) and Spitsbergen. Journal of Limnology 66 (Suppl. 1): 40-48. doi: 10.4081/jlimnol.2007.s1.40

Tsujimoto M, Imura S, Kanda H. 2016. Recovery and reproduction of an Antarctic tardigrade retrieved from a moss sample frozen for over 30 years. Cryobiology 72: 78-81. doi: 10.1016/j. cryobiol.2015.12.003

Van Rompu EA, De Smet WH. 1988. Some aquatic Tardigrada from Bjørnøya (Svalbard). Fauna norvegica. Serie A 9: 31-36.

Van Rompu EA, De Smet WH. 1991. Contribution to the freshwater Tardigrada from Barentsøya, Svalbard. (78³0’N). Fauna norvegica. Serie A 12: 29-39.

Van Rompu EA, De Smet WH. 1996. Freshwater tardigrades from Hopen, Svalbard ( $\left.76^{\circ} 31^{\prime} N\right)$. Fauna norvegica. Serie A 17: 1-9.

Vonnahme TR, Devetter M, Žárský JD, Šabacká M, Elster J. 2016. Controls on microalgal community structures in cryoconite holes upon high-Arctic glaciers, Svalbard. Biogeosciences 13: 659-674. doi: 10.5194/bg-13-659-2016

Węglarska B. 1965. Die Tardigraden (Tardigrada) Spitzbergens. Acta Zoologica Cracoviensia 11: 43-51.

Zawierucha K. 2013. Tardigrada from Arctic tundra (Spitsbergen) with a description of Isohypsibius karenae sp.n. (Isohypsibiidae). Polish Polar Research 34: 351-364. doi: 10.2478/popore-2013-0016

Zawierucha K, Coulson SJ, Michalczyk Ł, Kaczmarek Ł. 2013. Current knowledge of the Tardigrada of Svalbard with the first records of water bears from Nordaustlandet (High Arctic). Polar Research 32: 1-7. doi: 10.3402/polar.v32i0.20886

Zawierucha K, Cytan J, Smykla J, Wojczulanis-Jakubas K, Kaczmarek Ł, Kosicki JK, Michalczyk Ł. 2015a. Seabird guano boosts body size of water bears (Tardigrada) inhabiting the arctic tundra. Polar Biology 38: 579-582. doi: 10.1007/s00300014-1591-x

Zawierucha K, Kolicka M, Kaczmarek, Ł. 2016c. Re-description of the Arctic tardigrade Tenuibiotus voronkovi (Tumanov, 2007) (Eutardigrada; Macrobiotidea), with the first molecular data for the genus. Zootaxa 4196. doi: 10.11646/zootaxa.4196.4.2

Zawierucha K, Kolicka M, Takeuchi N, Kaczmarek Ł. 2015b. What animals can live in cryoconite holes? A faunal review. Journal of Zoology 295: 159-169. doi: 10.1111/jzo.12195

Zawierucha K, Smykla J, Michalczyk Ł, Goldyn B, Kaczmarek Ł. 2015c. Distribution and diversity of Tardigrada along altitudinal gradients in the Hornsund, Spitsbergen (Arctic). Polar Research 34: 1-11. doi: 10.3402/polar.v34.24168

Zawierucha K, Ostrowska M, Vonnahme TR, Devetter M, Nawrot AP, Lehmann S, Kolicka M. 2016d. Diversity and distribution of Tardigrada in Arctic cryoconite holes. Journal of Limnology 75: 545-559 doi: 10.4081/jlimnol.2016.1453

Zawierucha K, Vonnahme TR, Devetter M, Kolicka M, Ostrowska M, Chmielewski S, Kosicki JZ, 2016a. Area, depth and elevation of cryoconite holes in the Arctic do not influence Tardigrada densities. Polish Polar Research 37: 325-334. doi: 10.1515/ 
popore-2016-0009

Zawierucha K, Węgrzyn M, Ostrowska M, Wietrzyk P. 2017. Tardigrada in Svalbard lichens: diversity, densities and habitat heterogeneity. Polar Biology 40: 1385-1392. doi: 10.1007/ s00300-016-2063-2

Zawierucha K, Zmudczyńska-Skarbek K, Kaczmarek $€$, Wojczulanis-Jakubas K. 2016b. The influence of a seabird colony on abundance and species composition of water bears (Tardigrada) in Hornsund (Spitsbergen, Arctic). Polar Biology 39: 713-723. doi: 10.1007/s00300-015-1827-4

Editorial responsibility: Torkild Bakken.

This article is open-access and distributed under the terms of the Creative Commons Attribution 4.0 International license. This permits all non-commercial use, distribution, and reproduction in any medium, provided the original work is properly cited.

(http://creativecommons.org/licenses/by/4.0/). 